\title{
Photobiomodulation therapy improves multilineage differentiation of dental pulp stem cells in three-dimensional culture model
}

Ivana Maria Zaccara

Letícia Boldrin Mestieri

Maria Stella Moreira

Fabiana Soares Grecca

Manoela Domingues Martins

Patrícia Maria Poli Kopper 


\title{
Photobiomodulation therapy improves multilineage differentiation of dental pulp stem cells in three-dimensional culture model
}

\author{
Ivana Maria Zaccara, ${ }^{a}$ Letícia Boldrin Mestieri, ${ }^{a}$ Maria Stella Moreira, ${ }^{\mathrm{b}}$ Fabiana Soares Grecca, ${ }^{\mathrm{a}}$ \\ Manoela Domingues Martins, ${ }^{a}$ and Patrícia Maria Poli Kopper, ${ }^{\mathrm{a}, *}$ \\ ${ }^{a}$ Federal University of Rio Grande do Sul-UFRGS, School of Dentistry, Graduate Program, Department of Conservative Dentistry, \\ Porto Alegre, Brazil \\ bUniversity Ibirapuera-UNIB, School of Dentistry, São Paulo, Brazil
}

\begin{abstract}
Photobiomodulation therapy (PBM) has shown positive effects on stem cell differentiation in monolayer cell culture model, but little is known about its effect on three-dimensional (3-D) agarose gel culture. This study evaluated the PBM effect of human dental pulp stem cells (hDPSCs) differentiation and phosphatase alkaline activity (ALP) using an agarose 3-D model under different nutritional conditions. hDPSCs were characterized and seeded on a $0.3 \%$ agarose gel layer with different media (osteogenic, adipogenic, chondrogenic) and were assigned into four groups: control $10 \%$ fetal bovine serum (FBS), control $5 \%$ FBS, PBM $10 \%$ FBS, and PBM 5\% FBS. Irradiation was performed with continuous-wave InGaAIP laser, $660 \mathrm{~nm}, 100 \mathrm{~mW}, 3,3 \mathrm{~J} / \mathrm{cm}^{2}$, spot size $0.3 \mathrm{~cm}^{2}, 10 \mathrm{~s}$ of exposure time, and $1 \mathrm{~J}$ of energy per point with 6 -h interval between sessions. All groups were evaluated at 7 and 14 days. ALP assay was performed to analyze the deposition of mineralized tissue. At 7 days, PBM 5\% FBS group presented better stimulation in osteogenic and adipogenic differentiation compared with control. After 14 days, hDPSCs cultured in 3-D exhibited osteogenic, adipogenic, and chondrogenic differentiation; furthermore, compared to control, PBM significantly stimulated all differentiation processes $(p<0.05)$. It can be concluded that hDPSCs cultured in 3-D agarose associated to PBM could be a promising tool for tissue engineering applications. @ 2018 Society of Photo-Optical Instrumentation Engineers (SPIE) [DOI: 10.1117/1.JBO.23.9 .095001]
\end{abstract}

Keywords: dental pulp stem cells; low-level laser irradiation; photobiomodulation; bioactivity; differentiation.

Paper 180176RR received Mar. 22, 2018; accepted for publication Aug. 17, 2018; published online Sep. 10, 2018.

\section{Introduction}

Mesenchymal stem cells (MSCs) present important functions, such as capacity of self-renewal and development of tissues compatible with their origin. ${ }^{1}$ The interest in the isolation of human dental pulp stem cells (hDPSCs) has increased substantially in recent years and the teeth being considered a relatively rich source of MSCs. ${ }^{2}$ There are many markers to identify MSCs, including the STRO-1 surface marker, which is considered the main positive marker for MSCs and has the characteristic of not marking hematopoietic cells; CD44, a cell adhesion glycoprotein typical of mesenchymal cells; and CD146, a cell adhesion molecule. ${ }^{3}$ Several studies showed that hDPSCs could be characterized by a combination of these three markers together. ${ }^{1,3,4}$ In addition, it also recommended the analysis of differentiation in multilineage cells to confirm the stemness. ${ }^{5}$ The key to use stem cells is their ability to differentiate into various cell types, according to the stimulus received. MSCs present the potential of differentiation into osteoblasts, chondrocytes, adipocytes, muscle, and neural tissues, according to the microenvironment in which they are located. ${ }^{1}$

In this context, photobiomodulation therapy (PBM) is described as a form of light therapy that utilizes nonionizing forms of light sources, including lasers, LEDs, and broadband light, in the visible and infrared spectrum. It is a nonthermal

\footnotetext{
*Address all correspondence to: Patrícia Maria Poli Kopper, E-mail: patricia
} .kopper@ufrgs.br process involving endogenous chromophores eliciting photophysical (i.e., linear and nonlinear) and photochemical events at various biological scales. ${ }^{6}$ PBM could improve cell regeneration, stimulate cell growth, accelerate dentin regeneration after pulp exposure, increase cell metabolism, and promote tissue response. ${ }^{7,8}$ Additionally, PBM has been previously investigated and has been shown to promote biomodulation and increase the proliferation and differentiation of hDPSCs when applied in monolayer culture. ${ }^{9-11}$ It represents a beneficial impact on regenerative treatments, such as treatment of dental trauma or necrotic pulp with incomplete apex and periapical lesion, being used as a coadjutant treatment in protocols of repair and root development, accelerating these processes. ${ }^{9-12}$

Two systematic reviews demonstrated that irradiation should influence the differentiation effect when the energy density ranges from 0.5 to $4 \mathrm{~J} / \mathrm{cm}^{2}$ at wavelengths from 600 to $980 \mathrm{~nm}$, and that an energy density higher than $10 \mathrm{~J} / \mathrm{cm}^{2}$ could damage photoreceptors, reducing the biomodulation effect of laser. ${ }^{13,14}$ Moreover, according to these systematics reviews, the greatest values of proliferation or differentiation were obtained using high power density, low energy density, and short exposure time. ${ }^{13,14}$ Other studies verified a tendency of PBM to improve MSCs viability without deleterious effects; however, considering their method heterogeneity and the limited evidence, it is difficult to obtain a clear conclusion. ${ }^{15}$ 
Despite the important knowledge obtained from studies using monolayer cell culture, they do not accurately recreate the natural tissue architecture ${ }^{16}$ and some cell functions can be inhibited, thereby limiting their clinical application and relevance. ${ }^{17}$ Three-dimensional (3-D) cell culture analysis, which can better simulate in vivo the cellular conditions, can be a more physiologically relevant and predictive model, presenting greater stability and longer life expectancy than cells in monolayer culture. ${ }^{17}$ Moreover, the morphology of cells and intercellular signaling are more physiological in 3-D cultures than in monolayer culture, thereby promoting better knowledge about cellular function and differentiation in vivo. ${ }^{18}$ Thus, 3-D culture analysis seems to be more suitable for viability, cytocompatibility, and bioactivity assays. ${ }^{12,17}$

Additionally, in 3-D culture, when the PBM reaches biological tissues during irradiation, the laser light can be reflected, scattered, absorbed, or transmitted to the surrounding tissues. ${ }^{19}$ Furthermore, these interactions are also influenced by several specific laser characteristics, such as power output, oscillation mode (continuous wave or pulsed wave), or application mode (contact or noncontact). Among various 3-D culture models, the agarose gel (a polysaccharide of D-galactose and 3,6-anhydro-L-galactopyranose derived from the cell walls of red algae) presents favorable characteristics in relation to implantation: it is bioinert, nontoxic, and decreases the potential immune rejection in rats. ${ }^{20}$

Another important aspect is the positive influence of cultures under nutritional deficit conditions in the response of hDPSCs to PBM, improving cell growth. ${ }^{9}$ This happens because the nutritional deficit condition promotes an alteration of the redox state toward cell oxidation, and the cellular response to PBM is near optimal and stronger when potential redox is shifted. ${ }^{21,22}$ Moreover, cells with lower $\mathrm{pH}$ are considered more sensitive to the laser action than those with neutral $\mathrm{pH} .{ }^{9}$ The nutritional deficit aims to simulate a clinical situation in which the tissue is in a stress process. ${ }^{23}$ Therefore, the present study evaluated the PBM effect on osteogenic, adipogenic, and chondrogenic differentiation and phosphatase alkaline activity (ALP) of hDPSCs using an agarose gel 3-D model, under different nutritional conditions.

\section{Materials and Methods}

\subsection{Subjects}

The study was approved by the Institutional Review Board of the Federal University of Rio Grande do Sul, Brazil (CAAE 4545961 5.8.0000.5347). Human DPSCs were obtained from four permanent third molars with incomplete root formation, indicated for extraction due to orthodontic reasons, from two healthy patients aged 18 years old.

\subsection{Stem Cell Culture}

Freshly extracted teeth were immersed in alpha modification minimum essential Eagle's medium ( $\alpha$-MEM, Sigma-Aldrich) supplemented with $10 \%$ fetal bovine serum (FBS, Gibco, Life Technologies) and $1 \%$ penicillin and streptomycin (PenStrep, Gibco), and then transported to the laboratory for processing. After extraction, the apical papilla was removed and the pulp tissue was isolated from apical foramen using Hedstrom files (Maillefer, Switzerland). After pulp removal, it was sectioned into fragments of $\sim 1 \mathrm{~mm}^{3}$ and kept in supplemented $\alpha$-MEM at $37^{\circ} \mathrm{C}, 95 \%$ humidity, and $5 \% \mathrm{CO}_{2}$. The hDPSCs were obtained by explant culture in dish $(35 \times 10 \mathrm{~mm})$ containing $\alpha$-MEM. The culture medium was changed $24 \mathrm{~h}$ after pulp collection and at every $48 \mathrm{~h}$ to obtain explants of hDPSCs. When reaching the confluence ( $70 \%$ to $90 \%$ of cells) of the plate, the cells were trypsinized (Trypsin, Sigma-Aldrich) to the next passage.

\subsection{Stem Cell Characterization}

In the second passage (P2), the hDPSCs were analyzed by flow cytometry to confirm their stem cell nature. Briefly, an aliquot of cells was evaluated by staining for surface markers of MSCs (STRO1, CD146, and CD105) and for markers of hematopoietic stem cells (CD45 and CD14) (all from Santa Cruz Biotechnology). Additionally, the multilineage differentiation potential of hDPSCs, up to 14 days, was confirmed by culturing the cells in varied differentiation media:

(a) Osteogenic differentiation: $\alpha$ MEM supplemented with $1 \%$ penicillin and streptomycin, 10\% FBS, $100 \mathrm{nM}$ dexamethasone (Sigma-Aldrich, USA), $0.05 \mu \mathrm{M}$ ascorbate-2-phosphate (Sigma-Aldrich, USA) and $10 \mathrm{mM} \beta$-glycerophosphate (Sigma-Aldrich) ${ }^{24}$

(b) Adipogenic differentiation: $\alpha \mathrm{MEM}$ supplemented with $1 \%$ penicillin, $10 \% \mathrm{FBS}, 1 \mathrm{mmol} / \mathrm{L}$ dexamethasone, $5 \mu \mathrm{g} / \mathrm{mL}^{-1}$ bovine insulin (Sigma-Aldrich), $0.5 \mathrm{mmol} / \mathrm{L}$ isobutylmethylxanthine (Sigma-Aldrich), and $60 \mathrm{mmol} / \mathrm{L}$ indomethacin (Sigma-Aldrich), ${ }^{25}$ and

(c) Chondrogenic differentiation: $\alpha M E M$ supplemented with $1 \%$ penicillin and streptomycin, $10 \% \mathrm{FBS}$, $50 \mathrm{nmol} / \mathrm{L}$ ascorbic acid 2-phosphate, $6.25 \mathrm{mg} / \mathrm{mL}$ bovine insulin, and $10 \mathrm{ng} / \mathrm{mL}$ transforming growth factor-beta 1 (TGF- $\beta 1 /$ Sigma-Aldrich). ${ }^{26}$

\subsection{Three-Dimensional Culture and hDPSC Differentiation}

A pilot study was conducted to confirm the possibility of analysis of osteogenic, adipogenic, and chondrogenic differentiation of hDPSC in three-dimensional (3-D) culture. To obtain the 3-D culture, a $0.3 \%$ agarose solution (Sigma-Aldrich, St. Louis, MO) was prepared in $\alpha \mathrm{MEM}$ and $100 \mu \mathrm{L}$ of this solution were pipetted into each well of a 96-well plate (TPP, Trasadingen, Switzerland). After agarose solidification, $1 \times 10^{4}$ of hDPSCs (third passage) cultured in $150 \mu \mathrm{L}$ of $\alpha \mathrm{MEM}$ supplemented with $1 \%$ penicillin and streptomycin and regular $10 \%$ FBS were seeded in each well. During 14 days, the cells were maintained at $37^{\circ} \mathrm{C}, 95 \%$ humidity, and $5 \% \mathrm{CO}_{2}$ in osteogenic, adipogenic, and chondrogenic differentiation media, and after fixation, they were stained with alizarin red, oil red, and alcian blue, respectively. Homogeneous cell embedding in agarose was confirmed by light microscopy $(100 \times)$.

\subsection{Osteogenic, Adipogenic, and Chondrogenic Differentiation in 3-D Culture}

Table 1 shows the distribution of all groups according to PBM and nutritional status: regular (10\% FBS) or deficit (5\% FBS). The 3-D culture preparation and seeding of hDPSCs were performed as previously described in the pilot study. After $24 \mathrm{~h}$ of cell adhesion, the cells were induced to different differentiation 
Table 1 Experimental groups according to the PBM and nutritional condition (regular: $10 \%$ FBS or deficit: $5 \%$ FBS) for each differentiation analysis (osteogenic, adipogenic, and chondrogenic).

\begin{tabular}{lcc} 
Groups & PBM & Nutritional condition \\
\hline Control 10\% & - & Regular \\
Control 5\% & - & Deficit \\
PBM 10\% & + & Regular \\
PBM 5\% & + & Deficit \\
\hline
\end{tabular}

medium with $5 \%$ or $10 \%$ FBS according with the group. For that purpose, they were maintained in osteogenic, adipogenic, or chondrogenic media during 14 days. The medium was totally changed at every $48 \mathrm{~h}$ for 14 days.

\subsection{Photobiomodulation Therapy}

PBM was applied with a continuous-wave indium-galliumaluminum-phosphide (InGaAlP) diode laser (MM Optics Ltd., São Carlos, Brazil) using the following parameters: wavelength of $660 \mathrm{~nm}$, power of $100 \mathrm{~mW}$, spot size of $0.3 \mathrm{~cm}^{2}$, energy density of $3.3 \mathrm{~J} / \mathrm{cm}^{2}, 10 \mathrm{~s}$ of exposure time, $1 \mathrm{~J}$ of energy per point of application, 6-h interval, ${ }^{27}$ during 7 and 14 days consecutively, totalizing 28 and $56 \mathrm{~J}$ of energy in each point, respectively.

The laser probe was fixed perpendicular to each plate and in contact with the tissue culture plates. The cells were plated keeping empty wells between seeded wells, to minimize the unintentional dispersion of light between wells during laser application, and the output power of the equipment was tested using a power meter (Laser Check; MM Optics LTDA, Sao Paulo, Brazil). The control group was treated under identical conditions but with the laser device switched off.

\subsection{Alizarin Red Staining}

Cells were plated in triplicates according to the experimental groups described in Table 1. To evaluate the cells for their mineralized matrix synthesis potential, after 7 and 14 days, the cells were fixed in $10 \%$ formaldehyde solution (SigmaAldrich) washed with phosphate-buffered saline (PBS) $(3 \times)$ and mineralized extracellular matrix stained with $2 \%$ alizarin red (Sigma-Aldrich), ph 4.2, for $20 \mathrm{~min}$ (24). Quantitative analysis of the nodules was performed, as proposed by Hessle et al. ${ }^{28}$ For this, $1.0 \mathrm{~mL}$ of cetylpyridinium chloride (Sigma-Aldrich) was added to each well for $15 \mathrm{~min}$ for solubilization of nodules under agitation, followed by quantification in an ELISA reader (Thermo Fischer Scientific in, Waltham, Massachusetts) at 570$\mathrm{nm}$ wavelength. The measuring absorbance of a well containing only agarose was subtracted from the absorbance of test groups for the three differentiations below.

\subsection{Oil Red O Staining}

After culture in adipogenic medium, as previously described, staining with oil red $\mathrm{O}$ dye (Sigma-Aldrich) was performed to visualize lipid vesicles in the cells after 7 and 14 days of culture. The cells were fixed with $2 \%$ paraformaldehyde (Sigma-Aldrich) for $30 \mathrm{~min}$, washed with PBS, and stained with the dye diluted in PBS (6:4) for $1 \mathrm{~h}$. After this, the cells were washed with PBS and observed under a light microscope. Stained oil red O was also eluted with 4\% Nonidet P-40 (SigmaAldrich) in isopropanol ( $\mathrm{vol} / \mathrm{vol})$ for further quantification by spectrophotometry at $490 \mathrm{~nm} .^{29}$

\subsection{Alcian Blue Staining}

To evaluate the chondrogenic potential of cells, alcian blue (Sigma-Aldrich) dye staining was performed in the cultures after 7 and 14 days. The dye was prepared using $1 \mathrm{~g}$ of alcian blue dissolved in $100 \mathrm{~mL}$ of $3 \%$ acetic acid (Sigma-Aldrich). The cells were fixed with $2 \%$ paraformaldehyde (SigmaAldrich) for $30 \mathrm{~min}$, washed with PBS, and stained with the diluted dye in PBS (6:4) for $1 \mathrm{~h}$. Stained alcian blue was also eluted with $4 \%$ Nonidet P-40 (Sigma-Aldrich) in isopropa$\mathrm{nol}(\mathrm{vol} / \mathrm{vol})$ for further quantification by spectrophotometry at $490 \mathrm{~nm}^{29}$

\subsection{Alkaline Phosphatase Activity in 3-D Culture}

ALP enzyme was used as a marker of osteoblastic activity (Labtest Diagnostica, Lagoa Santa, MG, Brazil) and was measured in hDPSCs cultured in 3-D agarose after 7 and 14 days in all groups described in Table 1. The cells were lysed with TRIS $1 \%$ (Sigma-Aldrich) for $50 \mathrm{~min}$ at room temperature and the experiment was performed according to the manufacturer's instructions. The absorbance was measured in triplicate by 96-well plate (TPP) spectrophotometry at $590 \mathrm{~nm}$ in a spectrophotometer, the results were calculated and data were expressed as ALP enzyme activity (U/L)

\subsection{Statistical Analysis}

All experiments were performed in quadruplicate. Differences between test and control groups were analyzed by KruskalWallis followed by Dunn tests on the software GraphPad Prism 5 (GraphPad Software Inc., San Diego, California), considering a significance level of $5 \%(p<0.05)$.

\section{Results}

\section{1 hDPSCs Characterization}

The hDPSCs expressed typical levels of MSC surface markers (Fig. 1). The cultures expressed positivity for MSC markers (CD105, CD146, and STRO-1), whereas the hematopoietic cell markers (CD45 and CD14) were absent or minimally expressed. The percentage of positive cells for the CD105 was $98.8 \%$, for CD146 was $32.2 \%$, and STRO-1 was $3.9 \%$ for the hDPSC population. The percentage of cells for the CD45 was $0.3 \%$ and CD14 was $0.2 \%$ for the hDPSC population.

Moreover, those cells cultured in monolayer during 14 days showed a deposition of mineralized matrix as stained by alizarin red, lipid vesicles stained by oil red $\mathrm{O}$, and glycosaminoglycans deposits stained by alcian blue, indicating, respectively, the osteoblastic, adipose, and chondroblastic differentiation (Fig. 2). Thus, according to these results, the cells analyzed could be defined as hDPSCs. 

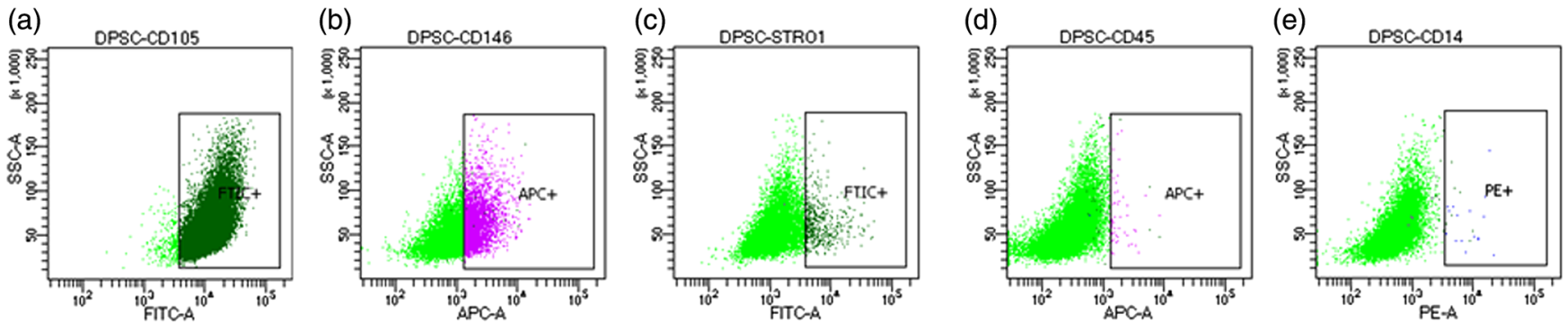

Fig. 1 Immunophenotyping of hDPSCs by flow cytometry. Cultures expressed positivity for MSC markers (a) CD105, (b) CD146, and (c) STRO-1, whereas the hematopoietic cell markers (d) CD45 and (e) CD14 were absent or minimally expressed.

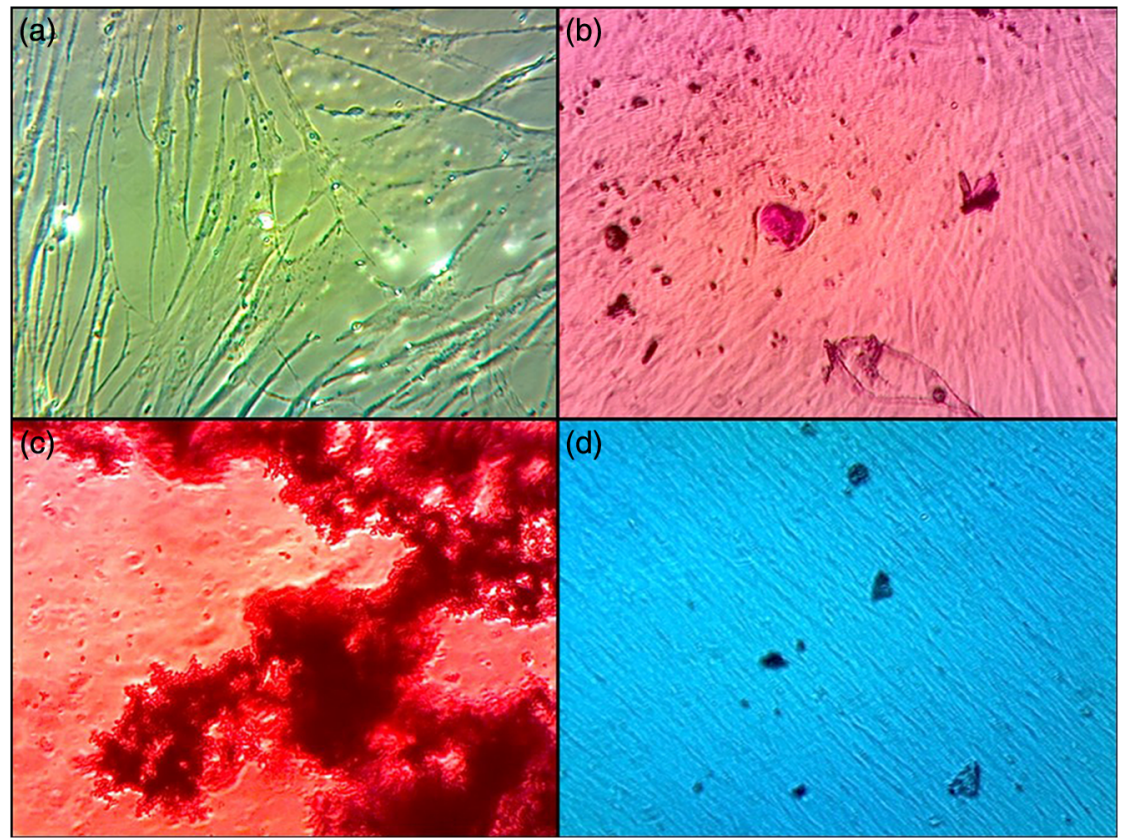

Fig. 2 Characterization of hDPSCs for their in vitro ability for osteogenic, adipogenic and chondrogenic differentiation. (a) hDPSCs cultured without induction of differentiation as negative control. (b) Calcium deposits stained with alizarin red. (c) Lipid vacuoles stained by oil red. (d) Alcian blue staining of glycosaminoglycans deposits (original magnification, $\times 100$ ).

\subsection{Photobiomodulation Therapy Promotes Increase in hDPSCs Differentiation in 3-D Agarose Model}

Initially, a pilot study was performed to evaluate the differentiation capacity of hDPSCs cultured in 3-D agarose model. As demonstrated in Fig. 3, hDPSCs cultured in 3-D after 14 days exhibited osteogenic [Fig. 3(a)], adipogenic [Fig. 3(b)], and chondrogenic differentiation [Fig. 3(c)]. These results indicated that agarose did not interfere with the differentiation properties of these stem cells and also in the absorbance measuring process.

Afterward, the effect of PBM on the ability of hDPSCs to differentiate in a 3-D culture model was evaluated (Figs. 4-6). PBM demonstrated positive effects, significantly stimulating both osteogenic, adipogenic, and chondrogenic differentiation processes. All irradiated groups significantly increased the process of the three types of differentiation after 14 days $(p<0.05)$.

Osteogenic differentiation was analyzed by a calcium deposition after 7 and 14 days of cell culture in 3-D and PBM treatment. After 7 days, only the PBM 5\% FBS presented significant increase in osteogenic differentiation compared to control 10\% FBS $(p<0.001)$ [Fig. 4(a)]. At 14 days, both groups that received PBM (10\% FBS and 5\% FBS) exhibited significantly increased mineralization compared to controls $(p<0.001)$ [(Fig. 4(b)].

The ALP assay in 3-D culture confirmed the results of osteogenic differentiation following the same trend in enzyme activity. At 7 days, the highest activity for ALP enzyme was observed in PBM 5\% FBS group compared with controls (10\% and 5\% FBS) [Fig. 4(c)] $(p<0.05)$. At 14 days, all PBM groups (10\% and 5\% FBS) presented significantly higher ALP compared to controls $(p<0.05)(10 \%$ and 5\% FBS $)$ [Fig. 4(d)].

Regarding the analysis of adipogenic differentiation after 7 days, a decreased capacity was observed in control 5\% FBS group compared to PBM groups (10\% FBS and 5\% FBS) [Fig. 5(a)]. However, at 14 days, all PBM groups (10\% FBS and $5 \%$ FBS) showed significant increase in lipid vesicles formation compared to control groups (10\% FBS and 5\% FBS) $(p<0.05)$ [Fig. 5(b)]. 
(a)

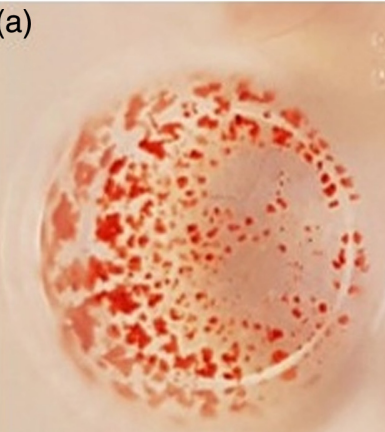

(d)

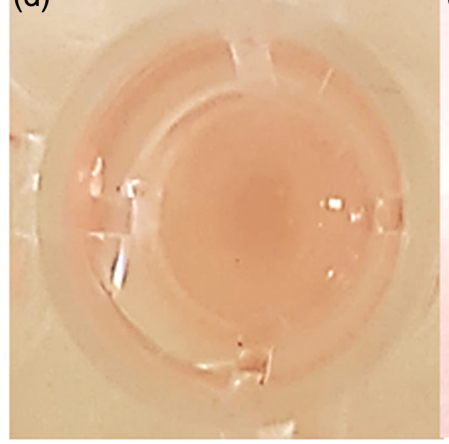

(b)

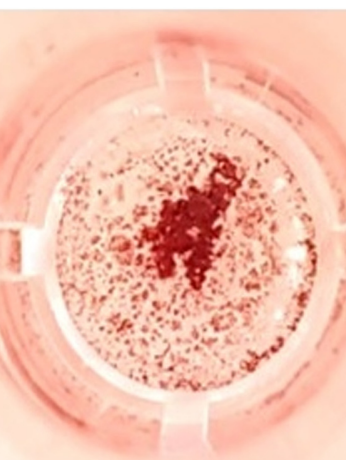

(e)

Fig. 3 Macroscopic aspect of controls and hDPSCs with osteogenic, adipogenic, and chondrogenic differentiation in 3-D agarose gel cultures. (a) Osteogenic differentiation, (b) adipogenic differentiation, (c) chondrogenic differentiation, (d) osteogenic control, (e) adipogenic control, and (f) chondrogenic control (original magnification, well diameter $=6.4 \mathrm{~mm}$ ). (c)

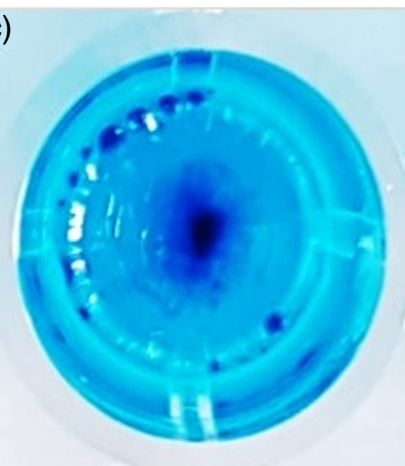

(f)

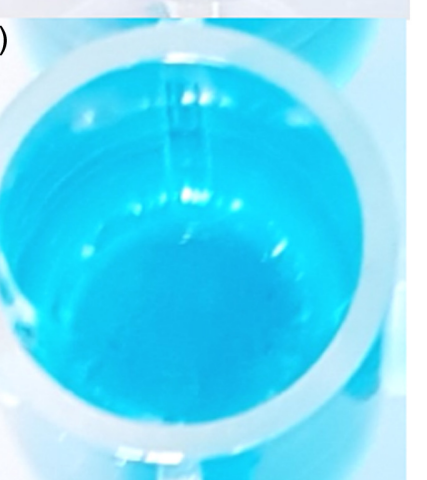

OSTEOGENIC DIFFERENTIATION
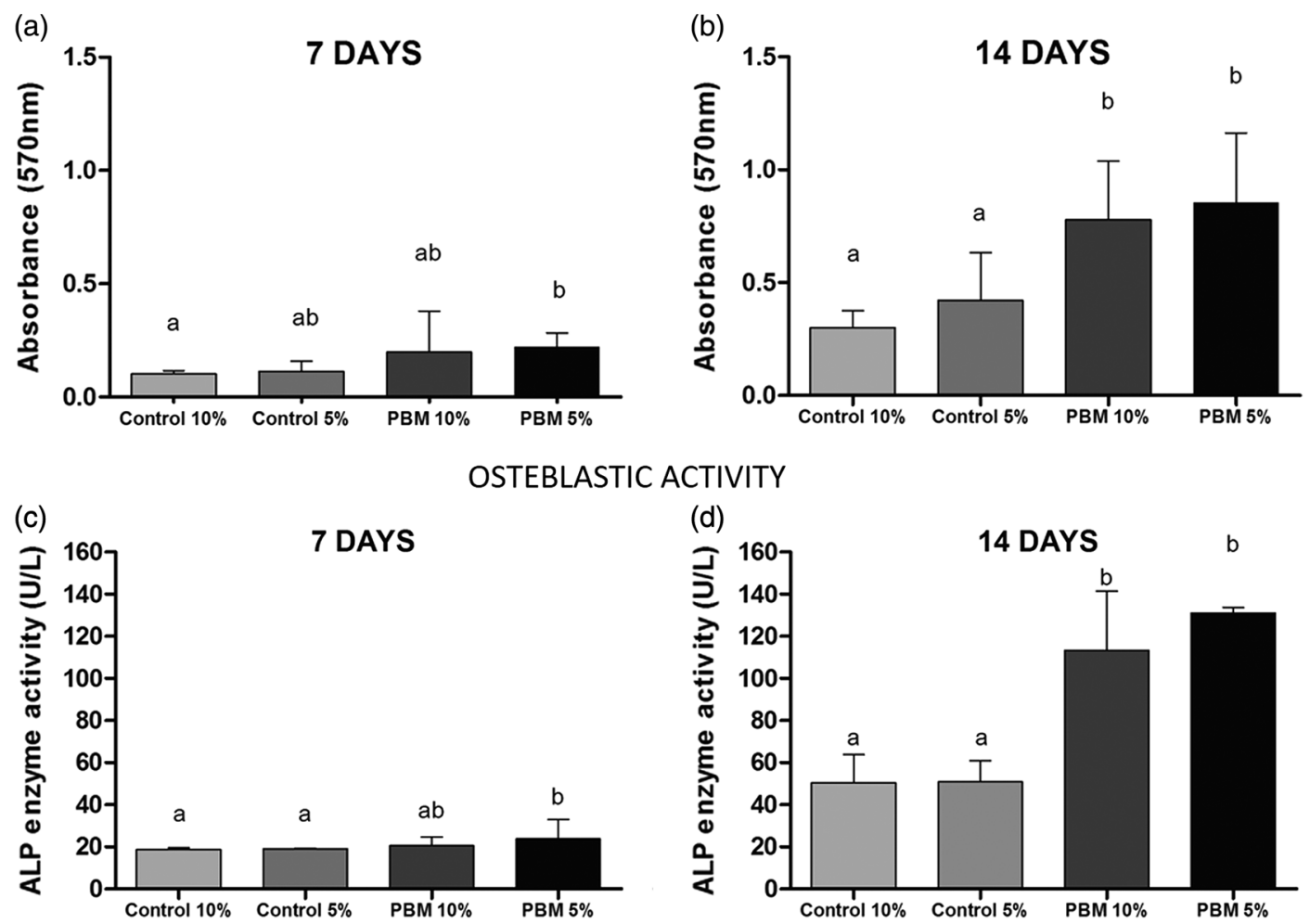

Fig. 4 PBM promoted an increase in (a and b) hDPSCs osteogenic differentiation and (c and d) osteoblastic activity. Bars with different letters represent significant differences between groups in each time point $(p<0.05)$. 
ADIPOGENIC DIFFERENTIATION

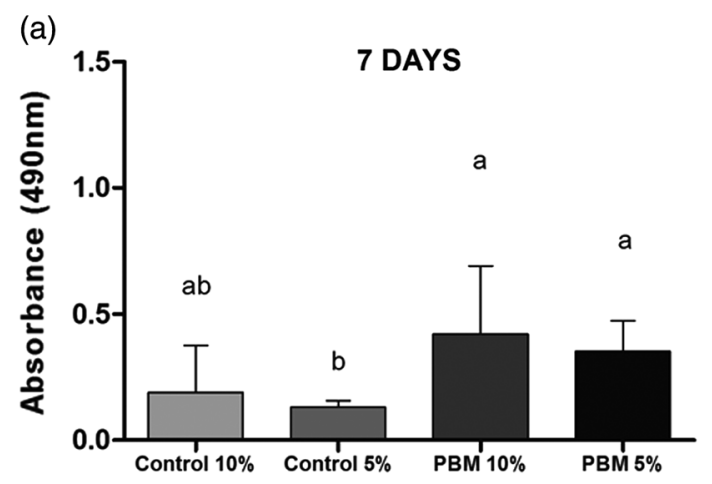

(b)

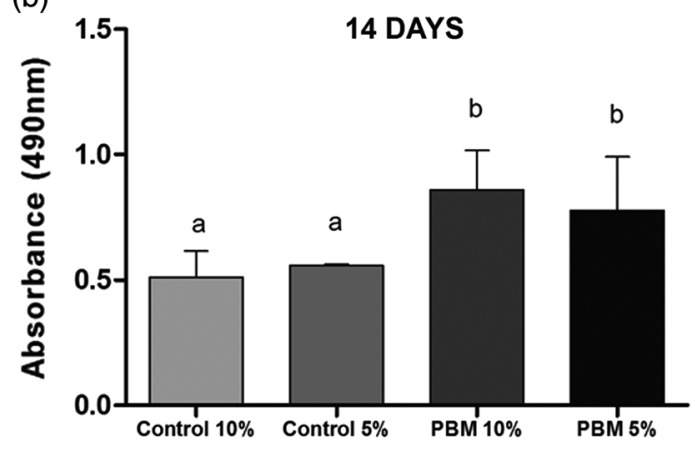

Fig. 5 Adipogenic differentiation of hDPSCs after (a) 7 and (b) 14 days of 3-D culture model. Bars with different letters represent significant differences between groups in each time point $(p<0.05)$.

\section{CHONDROGENIC DIFFERENTIATION}
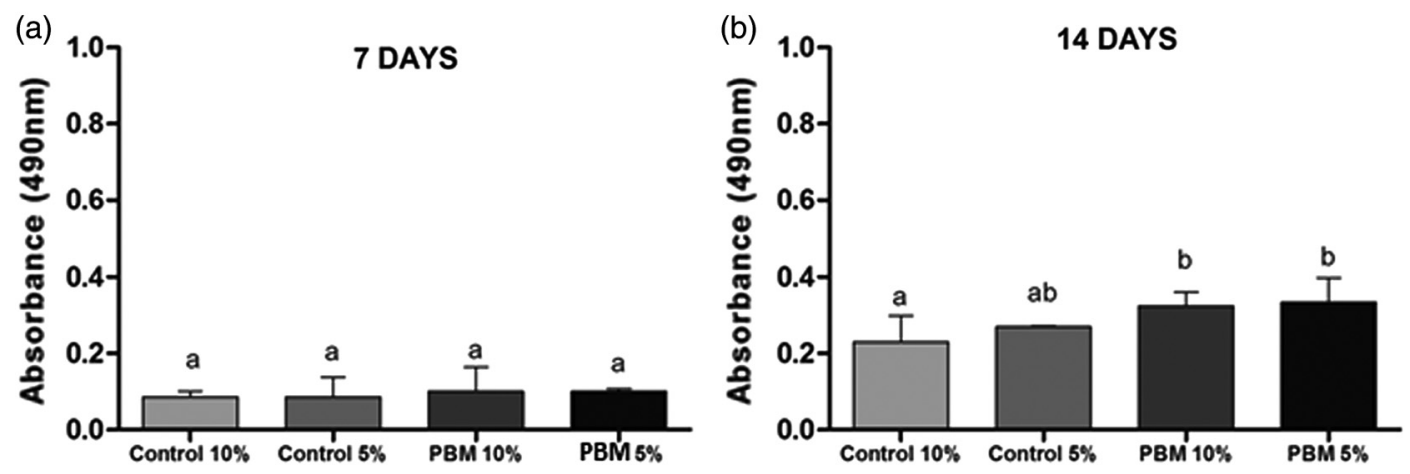

Fig. 6 Chondrogenic differentiation of hDPSCs after (a) 7 and (b) 14 days evaluated by the deposition of glycosaminoglycans. Bars with different letters represent significant differences between groups in each time point $(p<0.05)$.

Chondrogenic differentiation evaluated by a deposition of glycosaminoglycan was similar among groups at 7 days $(p>0.05)$ [Fig. 6(a)]. At 14 days, PBM promoted an increase in a deposition of these proteins compared only to the control $10 \%$ FBS $(p<0.05)$ [Fig. 6(b)].

\section{Discussion}

MSCs are characterized by their high proliferative potential, regenerative capability, and ability to differentiate in vitro into osteoblasts, chondrocytes, adipocytes, myocites, and neural cells according to the microenvironment in which they are located. ${ }^{1,30}$ A variety of tissues present MSCs that may be used in tissue engineering and regenerative medicine studies. ${ }^{1}$ Among them, the tooth is considered a rich source of these cells, identified by Gronthos et al. ${ }^{1}$ as DPSCs. Additionally, PBM enhanced the proliferation and differentiation of DPSCs in monolayer cell culture, which does not accurately recreate the natural tissue architecture. Within that context, the identification of factors that enhance the proliferation and/or differentiation of DPSCs is extremely important. Thus, the present study analyzed (a) the capacity of hDPSCs to differentiate in three lineages using 3-D agarose culture model and (b) the effect of PBM on the differentiation potential of hDPSCs in 3-D agarose culture model under different nutritional conditions. The PBM demonstrated positive effects, significantly stimulating the osteogenic, adipogenic, and chondrogenic differentiation process in 3-D model.
Initially, hDPSCs were obtained and characterized by the combination of two methods, multilineage differentiation potential (osteogenic, adipogenic, and chondrogenic) and by flow cytometry to assess the expression of STRO-1, CD44, and CD146 markers. These methodologies are corroborated by several studies that used the same markers of MSCs ${ }^{1,3,4}$ and also by three multilineage differentiation potentials. ${ }^{5,30}$ All these results were obtained using the monolayer culture (2-D) model that has been used as standard in several in vitro studies with MSCs; however, some authors appoint that this arrangement of cells does not mimic the cell-cell interaction of tissues. ${ }^{31}$ Currently, 3-D culture has become the best way to facilitate cell adhesion and proliferation, and it preserves the physiological function of tissue. ${ }^{16,18,32}$ Therefore, the 3-D culture model could mimic the natural tissue architecture, facilitating analysis of the differentiation potential, since it is influenced by the surrounding microenvironment. ${ }^{16,18,32}$ Then, we decided to investigate if hDPSCs maintain the capacity of osteogenic, adipogenic, and chondrogenic differentiation in three lineages using 3-D culture model. Some previous cell culture investigations used 3-D agarose gel model with different concentrations ranging from $0.9 \%$ to $3 \% .{ }^{33-35}$ Srikanth et al. ${ }^{35}$ demonstrated that a low concentration of agarose allows better understanding of the osteogenic differentiation when compared to higher concentrations. In the present study, we selected $0.3 \%$ agarose that was a favorable concentration for cell adhesion and differentiation with easy cell manipulation during change of media 
and analysis steps. Our results showed that hDPSCs maintained the capacity of multilineage differentiation using 3-D agarose culture model. These results are in accordance with some studies that demonstrated the synthetic and natural-based scaffolds, such as collagen sponges, chitosan, hydroxyapatite, polymer, biomatrix, or platelet lysate, have been used to load hDPSCs and showed that mineralized tissue can be formed when they are employed. ${ }^{32,36,37}$ Also, agarose has been considered a bioinert scaffold with nontoxic properties. ${ }^{20}$

$\mathrm{PBM}$ is an emerging technique in which exposure to low-level laser light (red and near infrared radiation) or LEDs stimulates cellular function by the activation of mitochondrial respiratory chain components, resulting in initiation of a signaling cascade that promotes activation of some signaling circuitries resulting in increased cellular metabolism and proliferation. ${ }^{21}$ Considering that PBM could allow a significant increase in viability, proliferation, migration, and differentiation of stem cells, the 3-D culture associated to its use may be a therapeutic opportunity in regenerative dentistry. ${ }^{11}$ Then, we tested the effect of PBM on the differentiation potential of hDPSCs in 3-D agarose culture model with different nutritional conditions.

The hDPSCs, in 3-D agarose-based model and irradiated at the energy density tested in the present study, presented early mineral deposition in the cultures (7 days after induction). Recently, Diniz et al. ${ }^{38}$ showed that PBM is able to improve cell survival of DPSCs encapsulated in an injectable and thermoresponsive cell carrier (Pluronic ${ }^{\circledR}$ F-127) loaded with recombinant human bone morphogenetic protein 4 (rhBMP4). In addition, PBM accelerated odonto/osteogenic differentiation of dental derived MSCs. ${ }^{38}$

Our main results showed that PBM (10\% FBS and 5\% FBS) promoted an increase in hDPSCs differentiation (osteogenic, adipogenic and chondrogenic), especially after 14 days, when compared with nonirradiated groups. These findings agree with previous investigations that analyzed the influence of PBM in osteogenic differentiation and ALP expression in monolayer culture using different irradiation parameters. ${ }^{38-40}$ By contrast, other authors also evaluated the PBM effect in hDPSCs using different 3-D cultures and observed that ALP activity increased until 7 days but gradually decreased until 14 days. ${ }^{12,32}$ These contradictory results could be justified by biomaterials used or PBM protocols, including the different time intervals between each application used at the referred studies: 1-day interval, 30 cycles of $30 \mathrm{~s}$ only at first day, or 3-day intervals, respectively. Herein, the 6-h interval was used because laser therapy exerts a dose-dependent effect on the biological responses and seems to have a cumulative effect at each new dose applied, and the best result of PBM has been observed in this time interval. ${ }^{27}$

Some studies attributed the biostimulative effect of PBM on osteoblasts to an increased expression of autocrine factors that regulate osteoblast proliferation and differentiation, such as type 2 bone morphogenetic protein (BMP-2) and TGF- $\beta 11^{12,39,40}$ These data suggest that PBM stimulates osteoblast differentiation in the initial proliferative and matrix synthesizing stages. Recently, an investigation using an in vivo model showed that PBM therapy allowed the stem cells to be influenced by the growth factors released from the root canal walls, resulting in cell differentiation and tissue formation. ${ }^{41}$ Also, molecular analyses indicated that red photobiomodulaton therapy in osteoblasts and hMSCs stem cells increased vinculin-rich clusters, osteogenic markers expression (Runx-2, alkaline phosphatase, osteopontin, Col I, OCN, DSPP, DMP1, and HSP27), and mineralized bone-like nodule structure deposition. ${ }^{42-44}$

Adipogenic differentiation was previously investigated to characterize the stem cells or evaluate some materials. ${ }^{18,25,30}$ However, until this moment, it was not used to quantify the lipid vesicle deposition induced by PBM. Similarly, in osteogenic differentiation and ALP activity, the adipogenic differentiation was increased in our study by PBM from 7 to 14 days $(p<0.05)$.

The association of PBM and chondrogenic differentiation was previously evaluated by Kushibiki et al. ${ }^{45}$ using a prechondrogenic activated T cell death (ATDC5) cells in monolayer culture. The authors observed that PBM enhanced the total collagen contents 14 days after low level laser irradiation $(p<0.01)$. Agreeing with them, the present investigation observed that hDPSCs induced chondrogenic differentiation in agarose gel, showing a significant increase in the deposition of glycosaminoglycans when PBM was used in comparison with the control $10 \%(p<0.05)$. The present findings about the PBM biostimulation on the adipocyte and chondrocyte expression genes and on the modulation of both differentiations must be further elucidated by new investigations.

With the intention to improve the effect of PBM on proliferation and differentiation, previous investigations demonstrated that a culture under nutritional deficit plays a positive influence on cell growth. ${ }^{9,43}$ Some studies evaluated the proliferation of DPSCs, human exfoliated deciduous teeth (SHEDs) and fibroblasts, respectively, under nutritional deficit of 5\% FBS in monolayer culture. ${ }^{9,23,46}$ They found that irradiated cells in nutritional deficit presented cell growth similar or higher than the control cells in ideal nutritional condition. No studies have evaluated the effect of PBM in hDPSCs differentiation under nutritional deficit (5\% FBS). Thus, the present study compared the osteogenic, adipogenic, and chondrogenic differentiation under nutritional deficit or regular nutrition in 3-D culture. Our results showed that in general, PBM improved the differentiation capacity of hDPSCs. In addition, deficit nutritional condition (5\% FBS + PBM) was associated to higher stem cell differentiation. This is a very significant discovery that can be justified by the fact that deficit condition alters the redox state toward oxidation of cells (oxidative stress) and the cellular response to PBM is near optimal and stronger when potential redox is shifted. ${ }^{21}$ Under conditions such as exposure to excessive or prolonged oxidative stress, the increase in ROS may reach a threshold level that triggers the opening of a mitochondrial channel favoring PBM effects. ${ }^{47} \mathrm{PBM}$ basic mechanisms have been described and are related to activation of cellular cytochromes that promote a cascade of events leading to increase in ATP production, reduction of oxidative stress, and initiation of secondary cell-signaling pathways. The overall results of PBM are the increase of energy metabolism, improvement of cell viability, and modulation of several cellular processes. ${ }^{44}$ Cells in nutritional deficit present higher oxidative stress than cells in normal condition (10\% FBS); therefore, they are more sensible to PBM stimulation. But, the mechanisms involved in the positive effect of PBM on hDPSCs differentiation need to be more deeply investigated. According to Hamblin, ${ }^{47}$ energy provided by PBM induces biomodulation by stimulating membranes, organelles, and modulation of oxidative stress of the target cell to restore its functions normality leading to hDPScs to have more differentiation or proliferation. 


\section{Conclusion}

PBM therapy positively influenced the differentiation process of hDPSCs cultured in 3-D agarose being a promising tool for tissue engineering applications.

\section{Disclosures}

No competing financial interests exist.

\section{References}

1. S. Gronthos et al., "Postnatal human dental pulp stem cells (DPSCs) in vitro and in vivo," Proc. Natl. Acad. Sci. U. S. A. 97(25), 13625-13630 (2000).

2. E. Piva, A. F. Silva, and J. E. Nör, "Functionalized scaffolds to control dental pulp stem cell fate," J. Endod. 40(4 Suppl), S33-S40 (2014).

3. S. C. Chen et al., "Location of putative stem cells in human periodontal ligament," J. Periodontal Res. 41(6), 547-553 (2006).

4. N. H. Lin et al., "Putative stem cells in regenerating human periodontium," J. Periodontal Res. 43(5), 514-523 (2008).

5. M. Dominici et al., "Minimal criteria for defining multipotent mesenchymal stromal cells. The International Society for Cellular Therapy position statement," Cytotherapy 8(4), 315-317 (2006).

6. J. J. Anders, R. J. Lanzafame, and P. R. Arany, "Low-level light/laser therapy versus photobiomodulation therapy," Photomed. Laser Surg. 33(4), 183-184 (2015).

7. H. Pretel, R. F. Lizarelli, and L. T. Ramalho, "Effect of low-level laser therapy on bone repair: histological study in rats," Lasers Surg. Med. 39(10), 788-796 (2007).

8. T. O. De Souza et al., "Phototherapy with low-level laser affects the remodeling of types I and III collagen in skeletal muscle repair," Lasers Med. Sci. 26(6), 803-814 (2011).

9. F. P. Eduardo et al., "Stem cell proliferation under low-intensity laser irradiation: a preliminary study," Lasers Surg. Med. 40(6), 433-438 (2008).

10. P. R. Arany et al., "Photoactivation of endogenous latent transforming growth factor- $\beta 1$ directs dental stem cell differentiation for regeneration," Sci. Transl. Med. 6(238), 238ra69 (2014).

11. I. M. Zaccara et al., "Effect of low-level laser irradiation on proliferation and viability of human dental pulp stem cells," Lasers Med. Sci. 30(9), 2259-2264 (2015).

12. A. Theocharidou et al., "Odontogenic differentiation and biomineralization potential of dental pulp stem cells inside Mg-based bioceramic scaffolds under low-level laser treatment," Lasers Med. Sci. 32(1), 201210 (2017).

13. K. M. Alghamdi, A. Kumar, and N. A. Mouss, "Low-level laser therapy: a useful technique for enhancing the proliferation of various cultured cells," Lasers Med. Sci. 27(1), 237-249 (2012).

14. A. N. Emelyanov and V. V. Kiryanova, "Photomodulation of proliferation and differentiation of stem cells by the visible and infrared light," Photomed. Laser Surg. 33(3), 164-174 (2015).

15. M. M. Marques et al., "Photobiomodulation of dental derived mesenchymal stem cells: a systematic review," Photomed. Laser Surg. 34(11), 500-508 (2016).

16. M. M. Cordeiro et al., "Dental pulp tissue engineering with stem cells from exfoliated deciduous teeth," J. Endod. 34(8), 962-969 (2008).

17. E. J. Silva et al., "Cytocompatibility of biodentine using a three-dimensional cell culture model," Int. Endod. J. 49(6), 574-580 (2016).

18. P. Vidyasekar et al., "Scaffold-free and scaffold-assisted 3D culture enhances differentiation of bone marrow stromal cells," In Vitro Cell. Dev. Biol. Anim. 52(2), 204-217 (2016).

19. F. Schwarz et al., "The impact of laser application on periodontal and peri-implant wound healing," Periodontology 2000 51, 79-108 (2009).

20. Y. Suzawa et al., "Biomineral/agarose composite gels enhance proliferation of mesenchymal stem cells with osteogenic capability," Int. J. Mol. Sci. 16(6), 14245-14258 (2015).

21. T. Karu, "Laser biostimulation: a photobiological phenomenon," J. Photochem. Photobiol. B 3(4), 638-640 (1989).

22. H. Friedmann et al., "A possible explanation of laser-induced stimulation and damage of cell cultures," J. Photochem. Photobiol. B 11(1), 87-91 (1991).
23. L. Almeida-Lopes et al., "Comparison of the low level laser therapy effects on cultured human gingival fibroblasts proliferation using different irradiance and same fluence," Lasers Surg. Med. 29(2), 179-184 (2001).

24. F. J. Rodríguez-Lozano et al., "Tissue engineering with dental pulp stem cells: isolation, characterization, and osteogenic differentiation," J. Craniofac. Surg. 23, e571-e575 (2012).

25. P. N. Taşlı et al., "Isolation and characterization of dental pulp stem cells from a patient with Papillon-Lefèvre syndrome," J. Endod. 39(1), 31-38 (2013).

26. L. Bernardi et al., "The isolation of stem cells from human deciduous teeth pulp is related to the physiological process of resorption," J. Endod. 37(7), 973-979 (2011).

27. D. T. Meneguzzo et al., "Influence of the fractioned irradiation energy in the phototherapy with low intensity laser on the growth of human dental pulp fibroblasts," Proc. SPIE 6846, 684601 (2008).

28. L. Hessle et al., "Tissue-nonspecific alkaline phosphatase and plasma cell membrane glycoprotein-1 are central antagonistic regulators of bone mineralization," Proc. Natl. Acad. Sci. U. S. A. 99(14), 94459449 (2002).

29. A. L. Sertié et al., "Effects of antipsychotics with different weight gain liabilities on human in vitro models of adipose tissue differentiation and metabolism," Prog. Neuropsychopharmacol. Biol. Psychiatry 35(8), 1884-1890 (2011).

30. M. F. Pittenger et al., "Multilineage potential of adult human mesenchymal stem cells," Science 284(5411), 143-147 (1999).

31. K. M. Yamada and E. Cukierman, "Modeling tissue morphogenesis and cancer in 3D," Cell 130(4), 601-610 (2007).

32. L. Abramovitch-Gottlib et al., "Low level laser irradiation stimulates osteogenic phenotype of mesenchymal stem cells seeded on a threedimensional biomatrix," Lasers Med. Sci. 20(3-4), 138-146 (2005).

33. H. Abuelba et al., "In vitro evaluation of curcumin effects on breast adenocarcinoma 2D and 3D cell cultures," Rom. J. Morphol. Embryol. 56(1), 71-76 (2015).

34. K. Park, Y. Nam, and Y. Choi, "An agarose gel-based neurosphere culture system leads to enrichment of neuronal lineage cells in vitro," In Vitro Cell. Dev. Biol. Anim. 51(5), 455-462 (2015).

35. L. Srikanth et al., "Gel based in vitro 3D model exploring the osteocytic potentiality of human CD34+ stem cells," Mol. Biol. Rep. 43(11), 12331242 (2016).

36. X. Yang et al., "Chitosan/collagen scaffold containing bone morphogenetic protein-7 DNA supports dental pulp stem cell differentiation in vitro and in vivo," J. Biomed. Mater. Res. Part A 00A (2011).

37. D. Abuarqoub, A. Awidi, and N. Abuharfeil, "Comparison of osteo/ odontogenic differentiation of human adult dental pulp stem cells and stem cells from apical papilla in the presence of platelet lysate," Arch. Oral Biol. 60(10), 1545-1553 (2015).

38. I. M. A. Diniz et al., "Photobiomodulation of mesenchymal stem cells encapsulated in an injectable rhBMP4-loaded hydrogel directs hard tissue bioengineering," J. Cell Physiol. 233(6) 4907-4918 (2018).

39. R. Medina-Huertas et al., "The effects of low-level diode laser irradiation on differentiation, antigenic profile, and phagocytic capacity of osteoblast-like cells (MG-63)," Lasers Med. Sci. 29(4), 1479-1484 (2014).

40. F. J. Manzano-Moreno et al., "The effect of low-level diode laser therapy on early differentiation of osteoblast via BMP-2/TGF- $\beta 1$ and its receptors," J. Craniomaxillofac. Surg. 43(9), 1926-1932 (2015).

41. M. S. Moreira et al., "In vivo experimental model of orthotopic dental pulp regeneration under the influence of photobiomodulation therapy," J. Photochem. Photobiol. B 166, 180-186 (2017).

42. A. Tani et al., "Red $(635 \mathrm{~nm})$, near-infrared $(808 \mathrm{~nm})$ and violet-blue $(405 \mathrm{~nm})$ photobiomodulation potentiality on human osteoblasts and mesenchymal stromal cells: a morphological and molecular in vitro study," Int. J. Mol. Sci. 19, 1946 (2018).

43. A. Ballini et al., "Osteogenic differentiation and gene expression of dental pulp stem cells under low-level laser irradiation: a good promise for tissue engineering," J. Biol. Regul. Homeost. Agents 29, 813-822 (2015).

44. A. C. F. Pedroni et al., "Photobiomodulation therapy and vitamin C on longevity of cell sheets of human dental pulp stem cells," J. Cell Physiol. 233, 7026-7035 (2018). 
45. T. Kushibiki et al., "Chondrogenic mRNA expression in prechondrogenic cells after blue laser irradiation," J. Photochem. Photobiol. B 98(3), 211-215 (2010).

46. C. Moura-Netto et al., "Low-intensity laser phototherapy enhances the proliferation of dental pulp stem cells under nutritional deficiency," Braz. Oral Res. 30(1), e80 (2016).

47. M. R. Hamblin, "Mechanisms and mitochondrial redox signaling in photobiomodulation," Photochem. Photobiol. 94(2), 199-212 (2018).

Ivana Maria Zaccara graduated in dentistry from the Federal University of Paraíba. She received her MSc degree in dentistry from the Federal University of Rio Grande do Norte, and her PhD in dentistry/endodontics from the Graduate Program in Dentistry of the Federal University of Rio Grande do Sul, Brazil.

Letícia Boldrin Mestieri graduated in dentistry from São Paulo State University, Brazil. She received her MSc degree in endodontics from São Paulo State University and her PhD in dentistry/endodontics from the Graduate Program in Dentistry of the Federal University of Rio Grande do Sul, Brazil.

Maria Stella Moreira received her postdoctoral at University of São Paulo, Brazil. She is full professor at Ibirapuera University and is researcher at Heart Institute, Clinical Hospital, São Paulo. She takes part in research group at University of São Paulo, working on translational research on regenerative endodontics, scaffolds, mesenchyme dental stem cells, and photobiomodulation for dental pulp tissue engineering. She is a member of the executive committee of World Federation for Laser Dentistry (South American Division).

Fabiana Soares Grecca is a full professor at the Conservative Dentistry Department, School of Dentistry, Federal University of Rio Grande do Sul, Brazil. She takes part in a research group at the Graduate Program in Dentistry of the Federal University of Rio Grande do Sul. Her personal research interests are the clinical and basic aspects of endodontic therapy.

Manoela Domingues Martins is a full professor at the Oral Pathology Department, School of Dentistry, Federal University of Rio Grande do Sul in Brazil. She is a postdoctoral researcher at the University of Michigan. Her personal research interests are the clinical and basic aspects of laser phototherapy in oral lesions and tissue regeneration.

Patrícia Maria Poli Kopper is a full professor at the Conservative Dentistry Department, School of Dentistry, Federal University of Rio Grande do Sul, Brazil. She takes part in a research group at the Graduate Program in Dentistry of the Federal University of Rio Grande do Sul. Her personal research interests are the clinical and basic aspects of endodontic therapy. 